\title{
Extended Bluetooth naming for empowered presence and situated interaction with public displays
}

\author{
Rui José ${ }^{1}$ and Francisco Bernardo ${ }^{2}$ \\ ${ }^{1}$ Departamento de Sistemas de Informação \\ Universidade do Minho. Campus de Azurem, \\ 4800-058 Guimarães, Portugal \\ rui@dsi.uminho.pt \\ ${ }^{2}$ Ubisign Tecnologias de Informação, Lda. \\ Edifício Olympus II. Rua Cidade do Porto, 79 \\ 4709-003 Braga, Portugal \\ info@ubisign.com
}

\begin{abstract}
The paradigm of proximity-based discovery and communication enabled by Bluetooth technology can be very relevant in Ambient Intelligence as an enabler for situated interaction. In this work, we explore the use of Bluetooth naming as a key driver for situated interaction around public displays. Our approach to the use of Bluetooth naming extends beyond self-exposure and introduces support for simple commands in the name that can trigger actions on the displays. Our specific objective is to evaluate the usability of this interaction technique and uncover any guidelines for its usage. We have conducted a study combining a trial in a public bar and a set of usability interviews. The results obtained confirm Bluetooth Extended Naming as an easily adoptable technique for situated interaction and suggest some recommendations to improve its effectiveness.
\end{abstract}

Keywords: Situated interaction, Bluetooth, Presence, Public displays.

\section{Introduction}

The paradigm of proximity-based discovery and communication enabled by Bluetooth technology is particularly well suited for situated interaction. In this work, we explore the use of Bluetooth naming as a key driver for situated interaction around public displays. Bluetooth devices have a user-defined name, created primarily for defining how Bluetooth devices present to each other, but which can be set and changed quickly. In the discovery process, these names become visible 
to nearby devices, enabling a simple proximate self-exposure mechanism, which has been enabling an increasingly strong culture around the social uses of Bluetooth naming [1]. Our approach to the use of Bluetooth naming extends beyond self-exposure and introduces Bluetooth Extended Naming as a technique in which the system can recognise parts of the Bluetooth device name as explicit instructions to trigger actions on the displays.

Bluetooth Extended Naming is necessarily a limited technique that does not aim to support any complex interaction dialogues with situated displays. There are several obvious limitations that may undermine its potential, such as the short size of Bluetooth names, limitations in text entry, and also the delay in the detection of name change updates introduced by the timings of the discovery process. It is however, in its technical simplicity, a promising technique for situated interaction. Firstly, because it has an extremely low entry barrier. Bluetooth is a widely available technology and setting new Bluetooth device names is normally a relatively simple task that can be accomplished with the base functionality of any mobile phone and without the need for any specialist software. This easy availability is of an huge importance in enabling social practices around the technology and represents a major difference to other sensing and interaction approaches that, albeit more sophisticated, require specific hardware or the installation of specialist software in personal devices. Secondly, the use of Bluetooth presence for situated interaction combines very well implicit and explicit forms of interaction, in fact blurring the distinction between them. Simply by having a discoverable Bluetooth device, people are already part of the situation and implicitly engaging with the system. This low bandwidth, but continuous, flow of presence information can be fundamental in the aggregation of situated content for the display and may act as an important catalyst for more explicit forms of interaction. This is what mainly differentiates interactions based on Bluetooth presence from interactions based on SMS, another widely available technology that is also very relevant for situated interaction with public displays.

\subsection{Research goals and overview}

Our research objective is to evaluate the effectiveness of Bluetooth extended naming as a technique for communicating commands for situated interaction around public displays. In the process, we also expect to uncover guidelines that may help to maximise the efficiency and potential uses of Bluetooth Extended Naming. In this study, we do not address the motivations and practices associated with the use of Bluetooth Extended Naming.

The methodology we chose combines a trial in a public setting and usability interviews. The trial provides important insight on the practical implementation of the technique and on its usability within the complex set of social phenomena that characterize situated interaction in a public setting. The interviews aim to inform the design with insight on the procedural mechanisms associated with this particu- 
lar use of Bluetooth naming. The results obtained from this study confirm Bluetooth Extended Naming as an easily adoptable technique for situated interaction and suggest some recommendations to improve its effectiveness.

In the remainder of this paper, we will start by reviewing in Section 2, the most relevant pieces of previous research that relate with our own work. In Section 3 we describe instant places, the system that served as an infrastructure for presence-based situated displays. In Section 4, we describe the trial we deployed in a bar and its results. In Section 5 we outline the main results from the usability interviews, and finally, in Section 6 we summarise our conclusions.

\section{Related Work}

The use of Bluetooth scanning has been extensively explored as a mechanism for sensing presence and uncovering all sorts of patterns, such as the familiarity level of the surrounding environment [2], the social situation [3], and more general large-scale reality mining [4]. In this work, we also build on the sensing possibilities enabled by Bluetooth discovery, but we take a different direction. Instead of intending to uncover information about an existing reality, our focus is on the use of Bluetooth naming as a means for empowered self-expression and as an enabler for situated interaction.

The work by Kostakos [5] in the cityware project is based on a platform for capturing mobility traces via Bluetooth scanning and has explored several ways of leveraging that information, including a set of in-situ visualizations providing people with information about current or recent Bluetooth presences. The system uses in-situ presence information as a way to generate content for a Facebook application that lets people associate physical co-presence information with their social network. We explore the opposite direction in that we aim to build on Bluetooth presence to generate situated content for local displays.

The Proactive displays [6] system explores the use of presence as a driver for situated interaction around public displays. The detection of nearby RFID tags triggers the display of profile information about the owner of the tag, promoting occasional encounters between people around the display. However, this type of approach requires a priori definition of individual profiles with associated data and assumes that everyone will be using a particular type of tag. Furthermore, people have a very limited role in the system, which is basically to move around and be detected.

The use of commands in short text messages is used by several on-line services that support SMS interfaces. The use of a picoformat [7] enables the codification of simple commands while satisfying the restrictions imposed by text input on mobile phones. Even though there are no standards for this, there some emerging SMS-based dialects to support interaction with mobile services, such as twitter nanoformats [8] and Dodgeball [9] . 


\section{Instant Places}

The instant places system displays on a public screen content that is directly or indirectly derived from Bluetooth presence information, including content derived from the presence of commands in Bluetooth names.

The system is composed by one or more Bluetooth enabled computers each connected to a public screen and linked to a central repository, as shown in Fig.1.

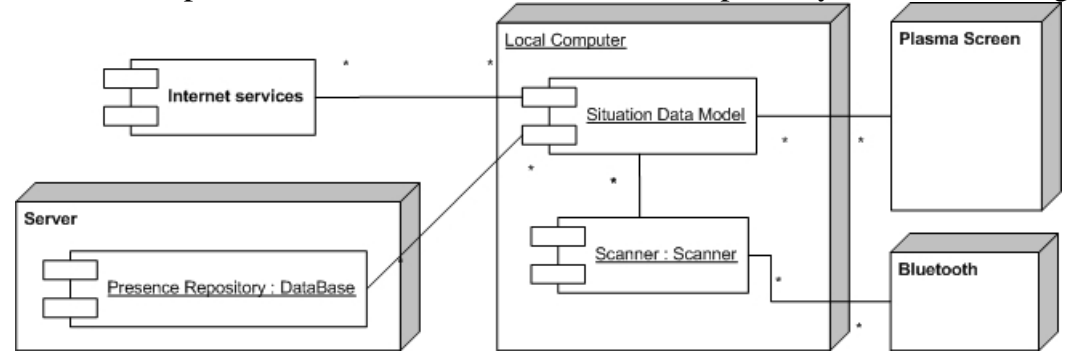

Fig. 1. Instant Places architecture

Information about nearby devices is periodically collected by a Bluetooth scanner and consumed by a situation data model that manages data about the place and present identities. A central repository is used to allow persistent identity information to be recognised across multiple sessions and also to combine information from pervasively distributed data sources, allowing for multiple screens in a large space to share the same presence view. The system does not need any a priori information about people, their profiles, permissions or groups, as all the information in the repository is entirely created from the history of presences.

Support for Bluetooth Extended Naming is an integral part of instant places. With every scanning operation, Bluetooth devices names are parsed in search for commands and their parameters. The syntax used is necessarily simple to comply with the constraints associated with Bluetooth device names. According to specifications, a Bluetooth device name can be up to 248 bytes and should be encoded based on UTF-8. This means that the maximum number of characters entered at the user interface level will actually vary with the type of characters being used and could be down to 82 characters. However, not all devices can be expected to handle more than the first 40 characters of the Bluetooth device name, and in many cases this may actually come down to the first 20 characters or even less. Additionally, text input on mobile devices suffers from restrictions associated with characters sets that may further restrain the syntax possibilities.

In the version of instant places that was used for this study, there were two types of commands, both defined as a command word followed by “:” and a set of comma separated parameters. The tag command associates multiple tags with the device, and is activated by including in the name the expression "tag:" followed by a comma separated list of tags, as in the following example "my device tag:punk,rock”. The Flickr command associate a Flickr user name with the device 
and is activated by including in the device name the expression "flk:" followed by the flickr user name, as in the following example "my device flk:JohnSmith". In both cases, these commands were intended to serve as hints for the display of photo streams obtained from the photo sharing website Flickr.

The central functionality supported by instant places is the visualisation on a public display of content that is situationally relevant and mainly driven by Bluetooth presence information, as shown in Fig. 2

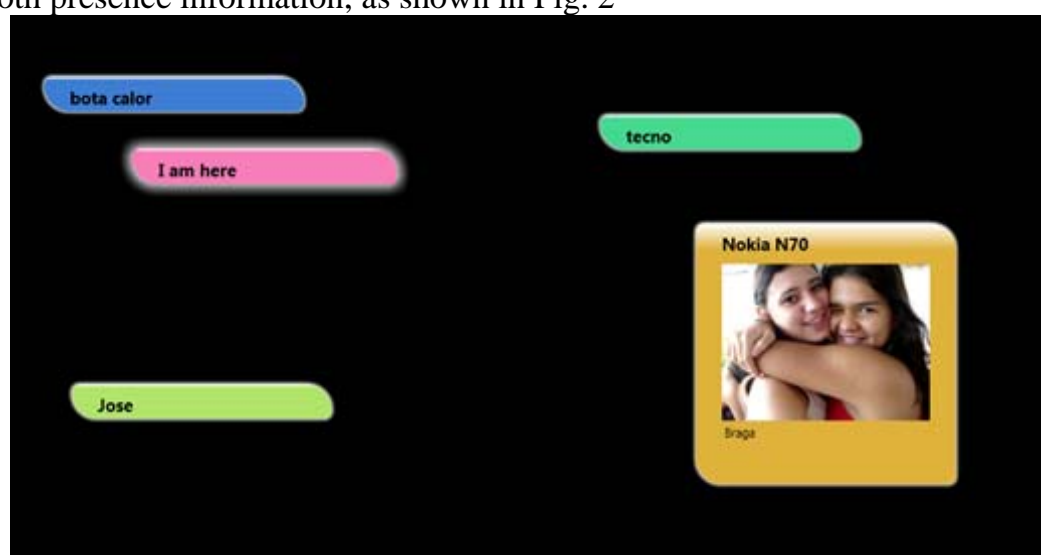

Fig. 2. Instant Places visualusation

The main driver for the visualisation is the real-time information about currently present devices and their names. The periodic scanning of Bluetooth devices generates a continuously changing flow of presence patterns that is visualised on the public display. Each discovered device is represented as a multivariate icon, in which the device name is clearly displayed. If the device name includes any commands, they are removed before the name is displayed. This use of Bluetooth names can be classified as an implicit form of interaction in the case of the person who unexpectedly finds his or her name on the display. However, it can quickly turn into an explicit form of interaction when that person changes the device name based on the new meaning created by the visualization on the screen.

These elements, per se, guarantee an important base level of content generation and implicit interaction. The dynamic patterns of Bluetooth presence provide an interesting and continuously changing element of situation awareness, and the public display of Bluetooth device names gives them a new meaning and empowers their use. However, they are limited in their ability to produce a continuous flow of enticing content, and that is where extended naming provides the extra level of functionality that invites people to more explicit and engaging forms of interaction. By including tag or $f l k$ commands in their device names, people can easily provide seeds that the system uses for selecting further content from Flickr. If a tag is used, the display presents photos tagged with that word. If a flickr user name is used, the display presents the respective photo stream. In both cases, photos are displayed directly on the device icon, which expands itself to create space 
for the photo display. This "situated mashup" manages to combine situated interaction with a global service to generate a continuously changing display of situationally relevant content.

\section{The trial at the Campus Bar}

The first part of this study was a public deployment of instant places. We chose a bar at the campus of University of Minho that is visited every day by several hundred people that come in for coffee or a quick snack, normally in small groups. There are several peak periods, with the busiest moment being at lunch time, when small meals are served Instant places visualisations were displayed using a 42" LCD screen that was already in the bar and is normally used for watching TV.

Prior to the public deployment of the system, we conducted a silent Bluetooth scanning for four weeks with the sole purpose of obtaining a neutral perspective of the local Bluetooth environment. When the system went public, we distributed leaflets with information about the project and instructions about the use of tags in Bluetooth names. The system used in this study was operational for 3 weeks.

The overall results of the trial indicate that the proposed techniques were easily and widely adopted as part of situated interactions around a public display. Table 1 compares Bluetooth usage parameters before and after the system was made public. We estimated the total number of visits to the bar based on sales numbers, and we collected from the logs information about how many unique device addresses and unique devices names were seen during these two periods.

Table 1. General Bluetooth usage patterns before and after instant places

\begin{tabular}{lll}
\hline & Silent Scanning & Instant Places \\
\hline Estimated visits & 7625 & 6526 \\
Unique devices & 365 & 460 \\
Unique names & 317 & 685 \\
\% visits with BT visible & $4.7 \%$ & $7.0 \%$ \\
Names per device & 0.9 & 1.5 \\
Names with commands & n.a & 112 \\
\hline
\end{tabular}

Even though no effort was made to recruit users, these numbers show a strong effect of the system on Bluetooth presence and naming patterns. There was a significant increase in the percentage of visitors that were visible for Bluetooth discovery (from $4.7 \%$ to $7.0 \%$ ), suggesting that a significant number of people made their device visible specifically for this purpose. Prior to instant places, there were more devices than device names, which can be justified by the existence of many devices using their default names. During the operation of the system, the average number of names per device raised to 1.5. Since there were no name changes during the initial period of silent scanning, it is sensible to conclude that all those 
changes were induced by the system. Regarding extended naming, there were 112 names that included tag commands. We analysed those names where tags were used to uncover any difficulties in the use of commands. The results are summarised in Table 2.

Table 2. Problems with the use of tag commands

\begin{tabular}{lll}
\hline Problem & Type & Occurrences \\
\hline Names with space after tag: & Syntax error 8 \\
Names with space between multiple tags & Syntax error 5 \\
Names without space before tag: & Syntax error 3 \\
Names only with tags & Ambiguous 6 \\
Names with tag: but no tag specified & Ambiguous 2 \\
Names with tag: repeated & Ambiguous 1 \\
\hline
\end{tabular}

There were 16 cases (14.2\% of those who tried to add tags to their name) with at least one syntax error that prevented the successful indication of tags. In 5 of those errors, the same name was observed with the correct syntax, indicating that after a first failed attempt, 5 people managed to somehow correct their mistakes and produce valid tags, lowering the failure rate to $9.8 \%$.There were also $9 \mathrm{am}$ biguous names that albeit not necessarily incorrect were not anticipated in our definition of the name rules. In 6 of them, the tag command was the name. This was not properly parsed because we were using the first word of the name as an identifier that was not considered for command parsing. There were 2 names without any tag specified, even though the "tag:" expression was present. Interestingly, this may have not been an error, but an emerging strategy for facilitating at a later point the recollection of the tag syntax, or reducing the input needed for adding new tags. One name had the "tag:" expression repeated, which in our current parser would ignore the second expression.

\section{Usability Interviews}

The second part of this study was a series of interviews with mobile phone owners to evaluate the performance in the execution of tasks related with Bluetooth naming and uncover any limitations or suggestions related with the use of Bluetooth Extended Naming. We conducted interviews with 40 participants (26 male and 14 female), aged between 15 and 28 who said to have had some type of Bluetooth usage before. The interview was divided in two distinct parts: a survey on Bluetooth naming practices that was answered by all the participants; and a set of 5 tasks related with Bluetooth naming that were executed by 12 of the participants. The tasks were executed with the participants own phones, and the objective was to measure performance and highlight any factors that could affect their execution. 
The first group of questions was about the visibility mode used in the Bluetooth device. $47 \%$ of the responds indicated always having their Bluetooth inactive or invisible. $41 \%$ responded that they occasionally had their device visible, $8 \%$ that they frequently had their device visible and $3 \%$ that they always had their device visible. The reasons for not being visible were most of all related with fears of attacks on the personal device, such as the possibility of infection by virus. Many people also mentioned a possible loss of privacy and saving energy (many respondents seemed to be aware of the consequence of Bluetooth on battery life). The reasons for activating Bluetooth and making it visible, were essentially sharing content (72\%) and device pairing (20\%). Some people specifically mentioned making use of "temporary visibility", a feature available in some devices that allows visibility to be activated only for a limited period of time.

Regarding Bluetooth naming practices, participants were asked how often did they change their Bluetooth device name. The vast majority answered that they rarely changed their name $(61 \%)$ or even that they had never changed their device name (31\%). Only 8\% reported changing their device name occasionally or frequently. On average, participants indicated being able to write device names with 31 characters on their mobile phone, with 16 being the minimum and 65 the maximum.

In the second part of the interview, participants were asked to perform the following 5 tasks:

- $\quad$ T1- Change the current activation state of their Bluetooth device.

- T2- Change their device name (new name had to be realistic)

- T3- Introduce a command in the device name (CMD:activate)

- T4-Introduce a command with two parameters (CMD:activate,num)

- T5-Introduce 2 commands, each with a parameter (CMD:activate CMD:num)

The graphics in Fig. 3 summarises the performance results.

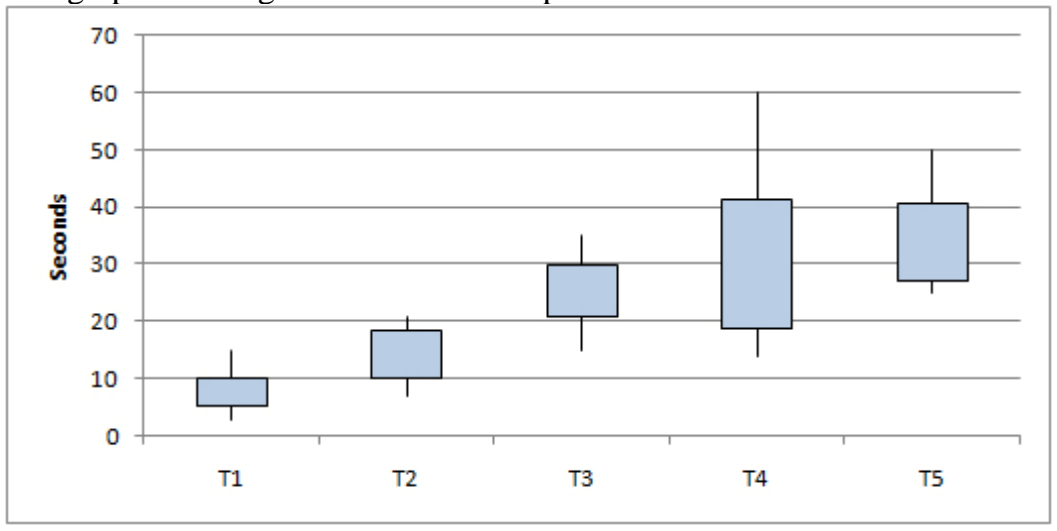

Fig. 3. Min, 1st Quartile, 3rd Quartile and Max times for task execution 
Results for tasks 1 and 2 indicate a generally good performance in the most basic operations of activating/deactivating Bluetooth (T1) and changing device name (T2). Particularly good results were obtained by participants with speed dial functionality already associated with Bluetooth activation/deactivation. These are tasks that can be easily and quickly achieved, even while on the move. The introduction of commands in device names (T3,T4 and T5) takes too long to be something that the average user would do without some disruption to other tasks. However, the average duration (about 30 seconds) is perfectly suitable for someone not immediately involved with other tasks, and these were tasks that people were doing for the first time. One of the participants was not able to complete tasks 3,4 and 5 because his device did not support whitespaces as part of the name.

Participants also made some suggestions on how to improve the performance of these tasks, such as using only lower case letters and replacing the use of the ":" character by the dot character ".”, more easily accessible on most mobile phones.

\section{Conclusions}

The overall results from our study show that there are no significant limitations for a widespread adoption of Bluetooth Extended Naming as a technique for situated interaction. The deployment of the trial has highlighted the high adoption rates that these techniques can have. Even without any active recruitment, a very significant number of people decided to try it. From those who have tried it, less than $10 \%$ seem to have been unable to achieve their purpose due to syntax errors. The results from the interviews also show that for most devices there are no significant limitations and that for most people, even without any previous training, the proposed operations can be executed within a periods of time that is appropriate for not too frequent operations.

We also intended to uncover guidelines that should be followed to maximise the efficiency and potential uses of Bluetooth Extended Naming. The results indicate that the major problem with the proposed syntax was the use of white spaces, suggesting the use of an alternative syntax that is more tolerant with the ambiguity that is normally associated with the use of white spaces. They also suggested the use of a character set that maximises the efficiency of text writing on mobile phones, such as a case insensitive syntax and the use of more commonly used characters such as "." as separator.

Regardless of these overall results, the individual experience will be largely determined by the mobile phone being used. Some devices still treat the Bluetooth name uniquely as a technical feature, resulting in cumbersome procedures for changing name and limitation on the length and format of the name itself that may restrict the appropriation of Bluetooth naming for other purposes, such as those studied in this work. However, severe limitations are not common and, what is more important, are not inherent to the Bluetooth standard. As the growing culture around Bluetooth naming continues to evolve these limitations are likely to disap- 
pear. These results should be considered by the manufacturers of Bluetooth devices that should aim to facilitate name writing and name change. The existence as part of the factory settings of speed dial options for Bluetooth configuration is a good indication that some device manufactures are attentive, but additional functionality, such as storing pre-defined names for easy selection (very much like changing profile) would be welcome in facilitating the use of these techniques.

\section{References}

[1] T. Kindberg and T. Jones, "“Merolyn the Phone”: A study of Bluetooth naming practices," presented at UBICOMP 2007 Ninth Inter Conf Ubiquitous Computing, Innsbruck, Austria, 2007.

[2] E. Paulos and E. Goodman, "The familiar stranger: anxiety, comfort, and play in public places," in Proceedings of the SIGCHI conference on $\mathrm{Hu}$ man factors in computing systems. Vienna, Austria: ACM, 2004.

[3] T. Nicolai, E. Yoneki, N. Behrens, and H. Kenn, "Exploring Social Context with the Wireless Rope," presented at 1st International Workshop on MObile and NEtworking Technologies for social applications (MONET'06), Montpellier, France, 2006.

[4] N. Eagle and A. Pentland, "Reality Mining: Sensing Complex Social Systems," Personal and Ubiquitous Computing, vol. 10, issue 4, 2006.

[5] V. Kostakos and E. O’Neill, "Capturing and visualising Bluetooth encounters.," presented at CHI 2008, workshop on Social Data Analysis, Florence, Italy., 2008.

[6] J. F. McCarthy, D. H. Nguyen, A. M. Rashid, and S. Soroczak, "Proactive Displays \& The Experience UbiComp Project," presented at First International workshop on Ubiquitous Systems for Supporting Social Interaction and Face-to-Face Communication in Public Places, Seattle, Washington, USA, 2003.

[7] A. Mabbett, C. Messina, M. Andy Stack, and A. Turner, "picoformats Microformats", http://microformats.org/wiki/picoformats (June 2008)

[8] G. Julio, "microblogging-nanoformats - Microformats", http://microformats.org/wiki/twitter-nanoformats (June 2008)

[9] Google Inc., "dodgeball.com :: mobile social software", http://www.dodgeball.com/glossary (June 2008) 